\title{
Le silence des fous chez Zola et Maupassant
}

«Les fous m'attirent. Ces gens-là vivent dans un pays mystérieux de songes bizarres, dans ce nuage impénétrable de la démence où tout [...] recommence pour eux $[. .$.$] en dehors de toutes les lois qui gouvernent les choses et régissent la pen-$ sée humaine " (Maupassant, 1979 : 874) - cette confidence de Maupassant aurait bien pu être signée Émile Zola. En effet, bien que le premier soit «malade d'esprit » (Gros, 1997 : 73) et l'autre se veuille extrêmement rationnel, l'œuvre des deux écrivains témoigne de la même fascination de la folie qui caractérise de nombreux écrivains de la seconde moitié du XIX ${ }^{e}$ siècle, époque dans laquelle, comme l'observent Philippe Hamon et Alexandrine Viboud,

[1]a maladie (chronique, professionnelle, héréditaire, handicap, infirmité, etc.) fait partie d'un champ thématique particulièrement riche, particulièrement exploité par le roman réaliste-naturaliste, celui de l'évocation du corps humain dans ses fonctionnements et dysfonctionnements psychiques et physiologiques. [...] À côté des maladies « traditionnelles », dont l'aspect héréditaire est à peu près toujours souligné [...], certaines comme la syphilis, la tuberculose ou les maladies psychiques (les «nerfs », la folie) occupent une place privilégiée dans la seconde moitié du siècle (Hamon, Viboud, 2008 : 83).

Dans plusieurs textes de Zola et de Maupassant, considérés, chacun à sa manière, comme deux grands écrivains de la folie, un des symptômes les plus manifestes des troubles mentaux est le silence. En effet, présentés en contradiction visible avec l'image stéréotypée d'un aliéné mental qui, incapable de se taire, produit constamment des sons - il gémit, hurle ou parle dans le vide -, les personnages des fous silencieux reviennent de façon récurrente dans les textes des deux auteurs. À travers leurs personnages, toujours « signifiants », en même temps dévoilant les zones d'ombre de l'individu et exemplifiant les antagonismes sociaux, Zola et Maupassant montrent la façon dont la maladie mentale - qu'elle prenne la forme d'un "détraquement ", d'une "fêlure "

Anna Kaczmarek-Wiśniewska - docteur ès lettres, maître de conférences à la Chaire de Culture et de Langue françaises de l'Université d'Opole. Adresse pour correspondance : Université d'Opole, Chaire de Culture et de Langue françaises, pl. Kopernika 11, 45-040 Opole, Pologne ; e-mail : akaczmarek@uni.opole.pl 
ou d'un simple « déséquilibre » dû à des causes variées -, ôte à lềtre humain une partie de sa personnalité. L'homme fou est surtout dépossédé de ce qui, étant donné sa nature de "parlêtre ", selon la définition lacanienne, constitue son privilège suprême : la capacité de communiquer par la parole. Nous avons écrit la façon, mais il serait peut-être plus approprié de parler des façons au pluriel, car le silence n'est jamais univoque :

[é]tudier le silence dans la littérature relève a priori du paradoxe. Dans ce magma de mots, dans ce "noirci" de pages, dans cette diversité de sens, [...] [1]e silence [...] est une notion complexe, bavarde et sa place dans le texte est aussi engageante qu'angoissante. Envisager ses formes, ses particularités, c'est considérer dès à présent que le silence est polysémique. Ces silences, donc, entretiennent des rapports ambivalents avec, d'une part, l'extériorité, et, d'autre part, l'intériorité du texte (Labeille, 2007).

Le présent article se propose d'analyser les différents sens du silence des fous qu'on peut observer dans plusieurs contes de Maupassant et dans quelques romans choisis de Zola. Impossibilité de parler suite à un traumatisme qui détache le protagoniste de la réalité (La folle de Maupassant), mutisme délibéré et obstiné d'un personnage enfermé dans un univers hallucinatoire (La chevelure du même auteur), effacement total de la capacité de s'exprimer suite à un détraquement mental anéantissant toute aptitude à penser (le personnage d'Adélaïde Fouque revenant tout au long du cycle zolien des Rougon-Macquart), ou encore le silence exalté d'un fanatique religieux qui ne se sent heureux que quand il plonge, sans une parole, dans la prière taciturne (La faute de l'abbé Mouret de Zola) : autant d'exemples d'un silence pathologique qui méritent, nous semble-t-il, une réflexion.

Si l'on admet que « [d]ans un premier temps, le silence se définit par la négative : absence de bruit, de son, quelque chose de vide » (Labeille, 2007), il devient évident que ce vide peut provoquer l'angoisse, la peur, voire l'obsession, capables de causer à leur tour une surexcitation pathologique, ou même un égarement des sens dont le fruit sont souvent des troubles hallucinatoires. C'est justement cet aspect du silence, perturbant, désorientant, voire désemparant l'individu, qui domine dans les contes et nouvelles de Maupassant désignés traditionnellement comme "contes de l'angoisse ». Et c'est également de ce type de silence, perçu comme un vide, une expérience qui se situe à la limite de la douleur physique, que parle Norbert de Varennes, le protagoniste de Bel-Ami, à Georges Duroy :

[...] vous ne savez pas ce que c'est que de vivre seul [...]. Une sorte de fièvre m'envahit, une fièvre de douleur et de crainte et le silence des murs m'épouvante. Il est si profond et si triste, le silence de la chambre où l'on vit seul. Ce n'est pas seulement un silence autour du corps, mais un silence autour de l'âme [...] (Maupassant, $1987: 301$ ).

Pour Maupassant, « dans la droite ligne d'une tradition humaniste attachée à la saisie par l'art des modalités de l'existence, l'écrivain est un psychologue de premier 
ordre " (Bury 2012). Ainsi, dans sa représentation du silence inquiétant et déroutant, " [l]'univers physique observable illustre la complexité de l'univers psychique, y compris et surtout, lorsqu'il se dérobe à l'emprise de la raison. La réalité d'un trouble n'est jamais appréhendée de façon abstraite " (ibid.). En effet, prenant en considération aussi bien les expériences personnelles de l'écrivain, sombrant progressivement dans la démence, que le regard qu'il porte sur la folie, Dominique Rougé constate que les textes de l'auteur représentent ce qu'on pourrait appeler la " folie réaliste » (Rougé, 2012 : 158); ce réalisme, épousant le célèbre style minimaliste des contes maupassantiens, fait éclater d'une manière particulièrement visible l'« inquiétante étrangeté " (Rougé, 2012 : 160) de l'expérience de la folie silencieuse.

Est-ce bien d'un "silence autour de l'âme " qu'il s'agit dans La folle, conte dont la brièveté augmente encore la cruauté ? Le narrateur de cette histoire de quelques pages à peine raconte avoir eu une voisine qui, à la suite de grands chagrins, demeurait muette et alitée pendant quinze ans, une sorte d'hystérie l'ayant comme paralysée :

[son] esprit s’était égaré sous les coups du malheur. Jadis, à l'âge de vingt-cinq ans, elle avait perdu, en un seul mois, son père, son mari en son enfant nouveau-né. [...] La pauvre jeune femme, foudroyée par le chagrin, prit le lit, délira pendant six semaines. Puis, une sorte de lassitude calme succédant à cette crise violente, elle resta sans mouvement, mangeant à peine, remuant seulement les yeux. [...] Que se passait-il dans cette âme désespérée? On ne le sut jamais ; car elle ne parla plus. [...] Pendant quinze années, elle demeura ainsi fermée et inerte (Maupassant $2007: 31-32)^{1}$.

Lors de la guerre de 1870, l'officier prussien qui habite chez elle croit qu'elle le nargue : il « s'imagina que la pauvre insensée ne quittait pas son lit par fierté, pour ne pas voir les Prussiens, et ne leur point parler, et ne les point frôler » $(F, 32)$. Se croyant bien malin, il veut l'obliger à se lever. Comme elle ne réagit point à ses interpellations, le regardant de " ses yeux vagues, ses yeux vides " $(F, 33)$, il se fâche et il la fait transporter avec son lit dans la forêt, persuadé que le froid la ramènera aussitôt chez elle. En effet, il s'agit d'un hiver où « il gelait à feindre les pierres» $(F, 32)$; qui plus est, la neige tombe jour et nuit pendant quelque temps, « ensevelissant la plaine et les bois sous un linceul de mousse glacée " $(F, 34)$. L'officier se trompe : la folle ne revient pas et on ne la revoit plus jamais. Au printemps, le narrateur, allé à la chasse, trouve un crâne humain et est persuadé que c'est celui de la folle qui a dû mourir de froid et être dévorée par les loups.

Comme c'est souvent le cas chez Maupassant, écrivain «banal ( Fonyi, 1984:5), il s'agit d'une tragédie insignifiante, d'une histoire dont le dramatisme même n'est pas tout à fait évident. Il est vrai qu'elle accumule plusieurs fatalités : celle des décès subis par la folle, celle de sa plongée dans la folie, celle de la méchanceté de l'officier qui exerce une cruauté inutile et bête, et celle enfin de l'hiver, qui, cette année-là, est

1. Désormais dans le texte: $F$, numéro de la page. 
particulièrement froid. Le dénouement reste incertain : rien ne donne l'assurance que la folle est vraiment morte, le narrateur lui-même ayant précisé que sa conviction que le crâne était bien celui de la folle n'est basée sur aucune preuve. Ce qui est pourtant indubitable, c'est que la cause de cette incertitude finale est bien le silence - d'abord celui de la folle qui est incapable (ou qui refuse) de parler même exposée à un danger mortel, puis celui qui s'installe sur la scène du drame avec la tombée de la neige et l'oubli de la folle disparue.

Le silence suggère aussi quelque chose d'incompréhensible, qui se dérobe à la perception : "Le "je ne sais quoi" qui est en jeu par la place du silence dans le texte reste insaisissable " (Labeille, 2007). C'est justement un fond de pensée qui reste insaisissable mais qui s'exprime par les symptômes d'une folie " pour ainsi dire palpable " (Maupassant 1992: 112) $)^{2}$ qui hante le personnage de La chevelure, exemple d'un " conte à tiroirs » composé d'une histoire peu signifiante dans laquelle est enchâssé un autre récit, le «vrai ». Dans un asile, un médecin discute avec le narrateur du cas de «folie érotique et macabre " $(C, 112)$ d'un malade qui demeure d'habitude tranquille et silencieux ; cet homme, qui paraît absent dans son propre corps, regardant le monde autour de lui "d'un œil fixe, vague et hanté ", est visiblement " ravagé, rongé par sa pensée, par une Pensée, comme un fruit par un ver » $(C, 111)$. Gardant le silence complet dans sa cellule "claire et sinistre ", il produit pourtant de temps en temps quelques sons sauvages, « un cri épouvantable, un hurlement de fureur impuissante et de désir exaspéré » $(C, 119)$. Le médecin fait lire au narrateur le journal intime du malade (c'est là que se trouve la "vraie » histoire). Selon ce document, l'existence paisible du jeune Parisien, collectionneur d'antiquités entièrement tourné vers le passé (il constate être "possédé par le désir des femmes d'autrefois »; $C, 113$ ), change radicalement lorsqu'un jour, il achète un meuble italien du XVII e siècle, qu'il ne se lasse pas de contempler et de manier. Dans un tiroir secret, il trouve une magnifique chevelure de femme, une longue natte blonde qui ne tarde pas à l'obséder : " Je la buvais, je noyais mes yeux dans son onde dorée " $(C, 118)$. Un beau jour, dans une hallucination de son imagination excitée, la femme porteuse de cette chevelure vient lui rendre visite, et il croit la tenir, la posséder : « Oui, je l'ai eue, tous les jours, toutes les nuits. Elle est revenue, la Morte, la belle Morte, l'Adorable, la Mystérieuse, l'Inconnue, toutes les nuits » $(C, 119)$. Mais, s'aventurant avec la chevelure à l'extérieur, dans la rue et au théâtre, l'homme est pris pour un fou et on l'enferme dans l'asile, en lui ôtant l'objet de son désir. Devant cette histoire, le narrateur reste stupéfait, « le cour battant de dégoût et d'envie » $(C, 120)$.

"Les personnages [de Maupassant] sont [...] prisonniers d'eux-mêmes, de leur peur ou de leur obsesssion» (Grosse, 1984 : 248). Le silence du fou épris de la chevelure est en effet une prison, une cellule, habitée, à part le malade lui-même, par sa bien-aimée imaginaire, par ses rêves macabres, par son désir désormais inassouvi : «Sa Folie, son Idée était là, dans cette tête, obstinée, harcelante, dévorante » $(C, 111)$,

2. Désormais dans le texte: $C$, numéro de la page. 
dit le narrateur. Cette prison est sournoisement bâtie par la chevelure trouvée qui, en apparence amicale, voire pleine d'affection, est en réalité hostile et oppressante. L'intrigue du conte confirme que "[1]e fantastique de Maupassant est sécrété [...] par des objets familiers, par des actions habituelles. Par une maison, un meuble, un aliment [...], qui, tout à coup, deviennent insupportables [...] et passent à l'attaque " (Fonyi, $1984: 12$ ). C'est dans le silence que le fou rumine dans son esprit ses idées et souvenirs, croyant les cacher devant ses « oppresseurs » de l'asile; et, paradoxalement, c'est le silence qui est son pire ennemi, l'enfermant dans l'indicible, dans l'impossibilité de verbaliser de manière compréhensible ce qui le ronge.

Des significations différentes sont attribuées au mutisme que gardent les personnages des fous zoliens. Viviane Alix-Leborgne distingue dans l'œuvre de Zola trois formes de folie : la folie douce (ou l'innocence, autrement dit le retardement mental), la folie lucide et celle furieuse, qui exige un internement immédiat du malade (AlixLeborgne, 2011). C'est comme à la charnière de la seconde et de la troisième forme que se situe le cas d'Adélaïde Fouque, dite Tante Dide, l'aïeule commune des Rougon et des Macquart. Décrite par le narrateur surtout dans le premier et le dernier volume, d'abord en tant que celle qui donne naissance à chacune des trois branches de la famille (une légitime et deux bâtardes), ensuite en tant que celle qui survit à tous, elle constitue l'alpha et l'oméga de toute la lignée sur laquelle son détraquement nerveux laisse une empreinte indélébile. Bizarre, déséquilibrée, en proie à des crises hystériques survenant dès sa jeunesse, elle sombre, octogénaire, dans la démence totale, sous l'influence d' " une affreuse douleur, un choc moral terrible " (Zola, $1977: 98)^{3}$ que fut la mort de son petit-fils Silvère lors des événements tumultueux de l'année 1851. Dans Le docteur Pascal, dernier volume de la saga, on la voit âgée de cent quatre ans, enfermée dans un asile; elle reste assise à regarder devant elle, n'ayant pas bougé depuis plusieurs années, "comme mangée par l'âge ", transformée en "squelette jauni » dont le cerveau est « ossifié » et qui n’a " plus que des yeux de vivants » (DP, 98-99), bien que ces yeux soient parfaitement vides de pensée et de sentiment quelconque. Ne parlant plus, riant ou pleurant parfois sans qu'on sache pourquoi ou balbutiant des mots incompréhensibles, elle a subi un «arrêt d'intelligence, un affaiblissement brusque, rendant toute réparation impossible " (ibid.), suite auquel elle est devenue " une démente calme, chez qui la folie $\mathrm{p}[\mathrm{eu}] \mathrm{t}$ rester indéfiniment stationnaire, sans amener la mort » (ibid.). Son petit-fils Pascal Rougon, médecin et savant, la caractérise en termes suivants :

[...] toute intelligence, tout souvenir parait aboli en elle. Le plus souvent, elle se tait ; parfois, elle a un flot bégayé de paroles indistinctes. Elle rit, elle pleure sans motif, elle est une chose que rien n'affecte... Et, pourtant, je n'oserais dire que la nuit soit absolue, que des souvenirs ne restent pas emmagasinés au fond... (DP, 99-100).

3. Désormais dans le texte: $D P$, numéro de la page. 
La démence de Tante Dide constitue la dernière étape d'un long processus, entamé par sa naissance d'un père fou et sa jeunesse marquée par des crises hystériques, accéléré par sa vie saccadée avec Macquart, braconnier et ivrogne, et venu à son point culminant avec la mort de ce dernier, puis celle de Silvère. Qu'elle a du tempérament, cela est indubitable, étant donné la violence de son amour pour Macquart ; or, ce tempérament ne s'exprime nullement par des flots de paroles, car Adélaïde a toujours parlé peu, et la mort de Macquart l'a figée dans un silence total, une douleur muette. $\mathrm{Si}$, pourtant, à cette étape-là on peut encore parler d'un choix qu'elle effectue de son propre gré - elle s'enferme dans sa maison et mène délibérément une vie monacale -, c'est d'un silence tout à fait différent qu'il s'agit après la mort de Silvère. Le cerveau de la vieille femme, endormi depuis des années, s’éteint définitivement et irrévocablement, elle perd la capacité de parler et semble ne pas entendre ce qui est dit autour d'elle. Il s'agit donc d'un silence sourd, un néant, un gouffre ténébreux qui, tout en protégeant Tante Dide du monde extérieur, l’emprisonne à jamais dans la nuit qui a submergé son esprit.

Le silence peut épouser aussi un sens positif en tant qu' " un prolongement de la parole, une source d'inspiration et un moment d'écoute » (Labeille, 2007). Tel semble être a prima vista le cas du silence solennel et mystique qui entoure Serge Mouret, protagoniste du cinquième volume du cycle zolien, La faute de l'abbé Mouret. Le jeune homme que le romancier a d'emblée condamné à incarner l'" affaiblissement » de la lignée, à être « eunuque, par le sang, par la race et l'éducation »(Ouvrard, 1986 : 66), cherche délibérément à s'effacer du monde, à vivre comme un ermite. Adolescent dévot et rêveur, il a été envoyé au séminaire dont il garde " toute l'ombre morte " (Zola, $1969: 30)^{4}$ dans son existence de prêtre, ce qui l'empêche de vivre une vie d'adulte ordinaire et le rend excessif dans ses passions dont la plus forte est une adoration fanatique de la Vierge, la seule figure féminine de son univers tout masculin. Le jeune prêtre a passé toute son adolescence et le début de sa vie d'adulte plongé dans la contemplation, s'entraînant à oublier son propre corps, ses sens et le monde environnant, rêvant à devenir un saint humble et tout entier tourné vers Dieu : « Il avait rêvé un désert d'ermite, quelque trou dans une montagne, où rien de la vie, ni être, ni plante, ni eau, ne le viendrait distraire de la contemplation de Dieu " (FAM, 30). Dans le village perdu des Artaud dont il est le curé, il vit ainsi « mourant à luimême, le dos tourné à la lumière ", désirant ardemment "n'être plus, [...] se perdre dans la souveraine blancheur des âmes " (FAM, 30), rester ignorant des passions humaines et des événements du quotidien pour ne pas perturber sa foi inébranlable. Les Artaud lui permettent de réaliser ce "rêve d'anéantissement humain ", de vivre « dans le sommeil des saints» (FAM, 30).

C'est Marie, ou plutôt la vision idéalisée et spiritualisée qu'il s'est fait d'elle, que le jeune prêtre contemple et adore en silence pendant de longues heures dans sa pauvre église de village où il retrouve ce dont il a besoin : la solitude, la concentration

4. Désormais dans le texte : FAM, numéro de la page. 
et l'oubli de la réalité. Ce culte muet mais extrêmement ardent aboutit à des fougues loin de la réalité, des moments où Serge semble se fondre dans l'élan passionné de sa foi, jusqu'à perdre conscience du lieu et du moment. Ses extases, ses croyances naïves et emportées auraient peut-être dû interpeller ses supérieurs au sein de l'Église ; or, il semble que personne ne se soit rendu compte du degré d'exaltation de ce jeune homme qui, s'il ne constitue pas un cas clinique de folie, est pourtant en proie à une inquiétante manie religieuse qui le détache non seulement de l'univers de sa paroisse, mais du monde des vivants en général.

Or, ce silence, en apparence pur et sublime, n'est point innocent, étant donné que, comme le montrent les expressions avec lesquelles le prêtre s'adresse à son idole " maîtresse si désirable ", " éternelle fleur du désir " (FAM, 115) -, son adoration pour la Vierge est en fait fortement sexualisée. En effet, les extases dans lesquelles Serge finit par s'oublier provoquent des pâmoisons dont les descriptions évoquent très nettement la jouissance sexuelle : il s'imagine "gravir un escalier de désir ", se sent " expirer " (ibid.) dans ces heures "de volupté divine " (FAM, 110). Dans cette optique, le silence s'investit d'un double rôle : catalyseur des élans du prêtre, il devient ainsi, en quelque sorte, leur résultat :

[c]ette jouissance [...] éloigne l'abbé de la sphère du logos, car il perd sa capacité langagière [...] ; ses prières à la Vierge deviennent un gazouillis incohérent, du rythme pur, nétant plus une suite de mots qui construisent un sens, mais une seule "parole sans cesse la même qui revenait » (Segarra, 1995 : 84).

L'histoire de Serge fait penser à celle du héros de La chevelure de Maupassant : ici et là, le silence du personnage véhicule une idée fixe, une obsession cachée. Il devient donc une sorte de trompe-l'œil, permettant aux deux hommes de dissimuler une activité fervente d'adorateur zélé, d'amant virtuel - ici, de la Vierge, et chez Maupassant, de la propriétaire fantasmagorique de la chevelure -, qui se servent, plus ou moins consciemment, de ce que Julia Kristeva qualifie de "non-langage ", d'une « communication sémiotique " qui ne coïncide pas avec la linguistique (Kristeva, 1983 : passim).

Il serait difficile de contester la justesse des propos de Véronique Labeille qui constate :

[i]l n'existe pas de définition unique du silence et ce dernier peut être conceptualisé par différents biais. Ainsi, il appert que la fonction communicative du silence entretient un rapport étroit avec le langage, mais le silence peut être aussi descriptif, ce sont les pauses, l'attente, ou relever de la description physique. [...] Le silence devient outil d'une rhétorique du sublime. Il dit, parle sur ce qui ne peut être articulé. Le silence incarne ce qui est irreprésentable. [...] Entre les silences suggestifs qui disent l'indicible et les silences de réflexion qui rendent compte de la difficulté de la verbalisation, sétend une partition de sons dont la polysémie n'est plus à prouver (Labeille, 2007). 
Deux hommes et deux femmes présentés dans les quatre textes analysés semblent relever de trois aspects de silence : renoncement (volontaire ? forcé ?) à la capacité de communiquer chez la folle de Maupassant ; effondrement du raisonnement et disparition des souvenirs chez Tante Dide; enfin, subterfuge permettant de s'adonner à sa monomanie dans le cas de l'homme amoureux de la chevelure et de l'abbé Mouret. De vrais aliénés ou considérés comme tels suite à leurs actions, les quatre personnages évoqués partagent pourtant tous une caractéristique : les conséquences du silence qu'ils gardent, tout remarquables qu'elles soient, ne sont jamais bienfaisantes. Maupassant se serait-il donc trompé en écrivant à propos des fous que " [e]ux seuls peuvent être heureux sur la terre, car, pour eux, la Réalité n’existe plus » (Maupassant, 1979: 874) ?...

\section{BIBLIOGRAPHIE}

Alix-Leborgne V. 2011. La folie chez Émile Zola et Hector Malot. Perrine, revue de l'Association des amis d'Hector Malot. En ligne, URL: https://www.amis-hectormalot.fr/revue-perrine/ revue-perrine-2011, consulté le 07/102017.

Bury M. 2012. Maupassant et les états d’angoisse. Colloque Fabula «L'anatomie du cœur humain n'est pas encore faite" : Littérature, psychologie, psychanalyse. En ligne, URL : http://www.fabula.org/colloques/document1646.php, consulté le 03/102017.

Fonyi A. 1984. Introduction : contes d'angoisse. In Maupassant G. de. Le Horla et autres contes d'angoisse. Paris. Flammarion.

Gros F. 1997. Création et folie. Une histoire du jugement psychiatrique. Paris. P.U.F.

Grosse Ch. 1984. Champs de lecture. In Maupassant G. de. Le Horla et autres contes d'angoisse. Paris. Flammarion.

Hamon Ph., Viboud A. (dir.). 2008. Dictionnaire thématique du roman de mours en France 1814-1914. Vol. 2. Paris. Presses de la Sorbonne nouvelle.

Kristeva J. 1985. Histoires d'amour. Paris. Gallimard.

Labeille V. 2007. Le silence dans le roman : un élément de monstration. Loxias 18. En ligne, URL : http://revel.unice.fr/loxias/index.html?id=1883, consulté le 13/10/2017.

Maupassant G. de. 1979. Madame Hermier. In Maupassant G. de. Contes et nouvelles. Vol. 2. Paris. Gallimard. coll. « Bibliothèque de la Pléiade ».

Maupassant G. de. 1987. Bel-Ami. Paris. Gallimard. coll. «Bibliothèque de la Pléiade ».

Maupassant G. de. 1992. La chevelure. In Maupassant G. de. Boule de Suif. Paris. Albin Michel.

Maupassant G. de. 2007. La folle. In Maupassant G. de. Contes de la bécasse. Paris. Albin Michel.

Ouvrard P. 1986. Zola et le prêtre. Paris. Beauchesne.

Rougé D. 2012. Écrire et lire la folie. Raconter le fou dans ses textes. Kraków. Wydawnictwo Naukowe Uniwersytetu Pedagogicznego.

Segarra M. 1995. Éros et transgression : la femme qui rit dans « La faute de l'abbé Mouret». Les Cahiers Naturalistes 69. 81-91.

Zola É. 1969. La faute de l'abbé Mouret. Paris. Fasquelle.

Zola É. 1977. Le docteur Pascal. Paris. Fasquelle. 
The silence of the mad in the works of Zola and Maupassant

ABSTRACT: It is difficult to define all the meanings and connotations of silence depicted in literary works. In the $19^{\text {th }}$ century, where the Realism and the Naturalism paid much attention to the study of both physical and mental illnesses, silence was considered as one of the distinctive signs of madness. The paper analyzes four examples of this phenomenon in selected Zola's and Maupassant's texts (novels and short stories) whose characters, all mad or maniac, embody various aspects of the silence regarded as a pathological condition of a human being.

Keywords: silence, madness, Zola, Maupassant. 